\title{
Alteration in methylation level at $11 \beta$-hydroxysteroid dehydrogenase type 2 gene promoter in infants born to preeclamptic women
}

\author{
Wensheng $\mathrm{Hu}^{1+}$, Xiaoling Weng ${ }^{2+}$, Minyue Dong ${ }^{1}$, Yun Liü${ }^{2,3}$, Wenjuan $\mathrm{Li}^{1}$ and Hefeng Huang ${ }^{1 *}$
}

\begin{abstract}
Background: Preeclampsia reduces placental expression and activity of $11 \beta$-hydroxysteroid dehydrogenase type 2 (HSD11B2), leading to an increase in fetal glucocordicoids. The latter has been proposed to be associated with low birth weight and high risk of metabolic diseases in later life of the offspring. This investigation aims to delineate the alteration in methylation levels at CpG sites of HSD11B2 promoter.

Results: Methylation levels of HSD9-2, HSD9-3, HSD23-2 and HSD23-3 and the mean methylation level were significantly lower in preeclampsia than in normal pregnancy $(P=0.002,0.031,0.047$ and 0.001 , respectively and $P<0.001$ in mean). The mean methylation level was significantly correlated with preeclampsia after the adjustment of birth weight, maternal age, gestational age at delivery and fetal gender $(r=0.325, P<0.001)$.

Conclusions: Preeclampsia reduced methylation level at fetal HSD11B2 promoter. A positive correlation existed between HSD11B2 promoter methylation and preeclampsia. Our findings suggest that the methyaltion status of HSD11B2 promoter is a potentially accessible biomarker for preeclampsia. However, further studies are required to address the mechanisms of thehypomethylation at HSD11B2 promoter and the significance of the hypomethylation in the development of metabolic diseases of the fetals born to preeclamptic women.
\end{abstract}

Keywords: Preeclampsia, Offspring, DNA methylation, Promoter, HSD11B2, Metabolic diseases

\section{Background}

Preeclampsia is a pregnancy-specific disease characterized by de-novo development of concurrent hypertension and proteinuria and sometimes progresses into a multiorgan cluster of varying clinical features. Complicating $2-8 \%$ of pregnancies, preeclampsia remains a leading cause of maternal and perinatal mortality and morbidity [1]. Furthermore, offspring of preeclamptic pregnancies are at the high risk of metabolic diseases in late life.

Worldwide, association studies between low birth weight and the subsequent development of common cardiovascular and metabolic disorders, including hypertension, insulin resistance, type 2 diabetes and cardiovascular disease death have been observed [2-4]. Relatively low birth weight is one of the characteristics of preeclampsia. Intrauterine

\footnotetext{
* Correspondence: huanghefg@hotmail.com

${ }^{\dagger}$ Equal contributors

'Women's Hospital, School of Medicine, Zhejiang University, 1 Xueshi Road, Hangzhou, Zhejiang Province 310006, China

Full list of author information is available at the end of the article
}

exposure to excessive glucocordicoids has been demonstrated to be one of the mechanisms leading to low birth weight. On the other hand, reduced placental expression and activity of 11ß-hydroxysteroid dehydrogenase type 2 (HSD11B2) which controls cellular concentration and the transmission of cortisol from the mother to the fetus have been documented in preeclampsia, which subsequently leads to the increased fetal glucocordicoids. The later phenomenon has been considered to be one of the mechanisms programming fetal risk of metabolic diseases in adulthood.

Animal models have suggested that methylation plays a critical role in placenta development, and alterations to its methylation pattern can lead to adverse placenta morphology and birth outcome [5]. In humans, the methylation status of promoter controls the gene expression and altered methylation levels at imprinted genes have been reported to be one of the mechanisms leading to increased risk of metabolic diseases in the offspring of preeclampsia [6]. However, few researches are available 
yet describing the alteration in methylation status at the promoter of HSD11B2 in the offspring of preeclamptic women.

The aims of this investigation were to clarify fetal methylation status at $\mathrm{CpG}$ sites of the promoter of HSD11B2 in preeclampsia and normal pregnancy, and discuss the possible role of alteration in methylation at the promoter of HSD11B2 in programming of the risk of metabolic diseases in the adulthood of the offspring of preeclampsia.

\section{Methods}

\section{Subjects}

Forty-three women of preeclampsia (25 of mild and 18 of severe) and 78 normal pregnant women were recruited in Women's Hospital, School of Medicine, Zhejiang University. The diagnosis of preeclampsia including severe preeclampsia and mild preeclampsia was defined as the previous study [7-9].

The control women had no sign of gestational complications and fetal distress and gave birth to healthy neonates of appropriate size for gestational age. All the participants are Han Chinese in origin.

The protocol of current investigation was approved by the Ethics Committee of Women's Hospital, School of Medicine, Zhejiang University and informed consents were obtained from all the participants.

\section{DNA methylation analysis}

Umbilical cord blood samples were collected in Ethylene Diamine Tetraacetic Acid (EDTA)-treated tubes at delivery for subsequent DNA extraction. Genomic DNA was isolated using the QIAamp DNA Blood Mini Kit following the standard protocol (QIAGEN, Hilden, Germany) and Thermo NanoDrop2000 (Thermo, Wilmington, USA) was used to detect 260/280 nm UV absorbance ratio and concentration. Bisulfite conversion of DNA was carried out using the Epitect Bisulfite Kit (QIAGEN, Hilden, Germany).

Quantitative methylation analysis of DNA was performed using MassARRAY EpiTYPER assays (Sequenom, San Diego, CA). At the promoter of HSD11B2, primers: I-F: 5' -aggaagagagTTTTTTTGTTTTTTAGAGTTTGG GG-3', I-R: 5'-cagtaatacgactcactatagggagaaggctAACA AAAACTAACCCAACCCTATCT-3'; II-F: 5' -aggaagag agGGTGGGTTATAAGTAATGGGAGATT-3', II-R: 5' cagtaatacgactcactatagggagaaggctCCACAAAACCTACCT AAAACAAAAA-3' were designed using Epidesigner (Sequenom, San Diego, CA; http://www.epidesigner.com). Polymerase chain reaction (PCR) amplification was performed using a $5 \mu \mathrm{l}$ reaction mixture with the following procedure: initial denaturation at $94^{\circ} \mathrm{C}$ for $4 \mathrm{~min}$, followed by 40 cycles of $95^{\circ} \mathrm{C}$ for $25 \mathrm{sec}, 58^{\circ} \mathrm{C}$ for $25 \mathrm{sec}, 72^{\circ} \mathrm{C}$ for $70 \mathrm{sec}$ and a final extension at $72^{\circ} \mathrm{C}$ for $7 \mathrm{~min}$. The PCR products were then incubated with shrimp alkaline phosphatase (SAP, Sequenom, San Diego, CA) at $37^{\circ} \mathrm{C}$ for $20 \mathrm{~min}$, followed by heat inactivation at $85^{\circ} \mathrm{C}$ for $5 \mathrm{~min}$. Transcription reactions were followed according to the manufacturer's standard protocol using T-cleavage assay kit (Sequenom, San Diego, CA). $20 \mu \mathrm{l} \mathrm{H} 2 \mathrm{O}$ and $6 \mathrm{mg}$ of Clean Resin (Sequenom, San Diego, CA) were added to the transcription products to remove bilvalent cation adducts. The samples were then sequenced on a MassARRAY analyzer (Sequenom, San Diego, CA) and analyzed with EpiTyper software (Sequenom, San Diego, CA). The amplicon comprised $6 \mathrm{CpG}$ sites at the HSD11B2 DMR in Human Genome 19 assembly - Chr16: 67,462,26767,462,481 and 67,464,230-67,464,394 (GRCh37/hg19), CpG site 1: 67,462,292; CpG site 2: 67,462,330; CpG site 3: 64,762,387; CpG site 4: 67,464,331; CpG site 5: 67,464,368; CpG site 6: 67,464,389.

\section{Statistical analysis}

The Kolmogorov-Smirnov tests were used to evaluate the distribution of data. Student t-tests were used for the comparison of continuous data between groups. Chisquare test was used for the analysis of categorical data. Linear mixed model analysis was used for the relationship of methylation level with the presence of preeclampsia, birth weight, maternal age, gestational age at delivery and fetal gender. Multivariate linear regression was used to evaluate the correlation of mean methylation level with birth weight, maternal age, gestational age at delivery and fetal gender. SPSS statistical package (Statistical Analysis System, Chicago, IL) was used for the data analysis. Values of $\mathrm{P}<0.05$ were considered to be statistically significant.

\section{Results}

As shown in Table 1, no significant differences between preeclamptic and control pregnancies were observed

Table 1 Characteristics and average methylation levels of study population

\begin{tabular}{lccc}
\hline & Normal pregnancy & Preeclampsia & P value \\
\hline TOTAL N & 78 & 43 & \\
Maternal age (y) & $28.56 \pm 3.36$ & $31.67 \pm 4.68$ & 0.356 \\
Gestational age (y) & $38.77 \pm 1.40$ & $36.11 \pm 3.16$ & 2.904 \\
Birth weigh (g) & $3287.82 \pm 393.30$ & $2626.98 \pm 860.60$ & 0.014 \\
HSD9_CpG_1 & $0.13 \pm 0.027$ & $0.12 \pm 0.031$ & 0.279 \\
HSD9_CpG_2 & $0.10 \pm 0.019$ & $0.09 \pm 0.018$ & 0.002 \\
HSD9_CpG_3 & $0.41 \pm 0.048$ & $0.39 \pm 0.056$ & 0.031 \\
HSD9 & $0.22 \pm 0.026$ & $0.21 \pm 0.030$ & 0.053 \\
HSD23_CpG_1 & $0.16 \pm 0.036$ & $0.16 \pm 0.036$ & 0.789 \\
HSD23_CpG_2 & $0.17 \pm 0.096$ & $0.13 \pm 0.031$ & 0.047 \\
HSD23_CpG_3 & $0.15 \pm 0.064$ & $0.11 \pm 0.048$ & 0.001 \\
HSD23 & $0.16 \pm 0.051$ & $0.13 \pm 0.029$ & 0.007 \\
\hline
\end{tabular}


with regard to age, fetal gender. There was no significant difference in gestational age at delivery. The neonatal birth weights were significantly lower in differences in preeclampsia than normal pregnancy.

The methylation levels were detected at $6 \mathrm{CpG}$ sites of HSD11B2 promoter. There were no significant differences at any of these $6 \mathrm{CpG}$ sites between male and female infants in either normal pregnancy $(\mathrm{P}>0.05$ for all) (Table 2) or preeclampsia ( $\mathrm{P}>0.05$ for all) (Table 1). Then, data of male and female infants were pooled. The methylation levels at sites HSD9-2, HSD9-3, HSD23-2 and HSD23-3 of HSD promoter were significantly lower in preeclampsia than in normal pregnancy $(\mathrm{P}=0.002,0.031$, 0.047 and 0.001 , respectively) (Figure 1), but the methylation levels at site HSD9-1 and HSD23-1 did not significantly differ $(P=0.279$ and 0.789 , respectively, Table 2$)$ (Figure 2). The methylation levels of HSD11B2 promoter were not significantly different between mild and severe preeclampsia ( $\mathrm{P}>0.05$ for all, Table 3$)$.

The mean methylation levels at the HSD11B2 promoter were significantly different between normal pregnancy and preeclampsia $(\mathrm{P}=0.007$, Table 1$)$. The mean methylation level was significantly lower in preeclampsia than normal pregnancy $(\mathrm{P}<0.001)$. There was no significant difference in mean methylation level between mild and severe preeclampsia $(\mathrm{P}=0.672)$ (Figure 2).

The mean methylation level was significantly correlated with preeclampsia after birth weight, maternal age, gestational age at delivery and fetal gender were adjusted $(\mathrm{r}=0.325, \quad \mathrm{P}<0.001)$, but not significantly correlated with birth weight $(P=0.165)$, maternal age $(P=0.778)$, gestational age at delivery $(\mathrm{P}=0.064)$ and fetal gender $(\mathrm{P}=0.801)$.

\section{Discussion}

HSD11B2 is abundantly expressed in placenta and controls intracellular concentration of cortisol as well as the transition of cortisol from the mother to the fetus. Placental expression and activity of HSD11B2 is reduced in preeclampsia with comparison to normal pregnancy $[8,9]$. Diminished 11b-HSD11B22 activity which allows for more than $10-20 \%$ passage of maternal glucocorticoids leads to increased fetal blood cortisol levels in preeclampsia [10-12]. Glucocorticoids, such as cortisol and corticosterone control fetal and pre- and postnatal development evolving the term glucocorticoid programming. This is thought to affect the brain, the hypothalamic-pituitary axis, the blood pressure regulatory systems, the heart, glucose-insulin homeostasis and metabolism, the pancreas and fat tissue [13,14]. Increased level of fetal glucocorticoids has been associated with low birth weight and subsequent increased risk of metabolic diseases in adulthood in humans and animal models [15-18].

During development, 11b-HSD11B2 is highly expressed in fetal tissues including kidney, lung, gut and notably brain [19-21]. However, the alteration in fetal HSD11B2 expression and activity has never been delineated yet in preeclampsia. In this investigation, we revealed for the first time the reduced fetal methylation at CpG sites of HSD11B2 promoter in preeclampsia, implying the expression of HSD11B2 is likely to be increased. Although it was reported that HSD11B2 was expressed in fetal kidney in human from early gestation, the expression and activity of HSD11B2 were determined in neither fetal kidney nor other tissues at birth. Unpublished data from our laboratory showed the expression of HSD11B2 in fetal kidney of X-induced preeclampsia model is decreased, whereas intrauterine growth restriction (IUGR) increased the methylation at CpG sites of HSD11B2 promoter in animal model. Those findings imply the complexity in the effects of pregnancy complications on fetal HSD11B2 expression and activity.

Interestingly, our present finding of a hypomethylation level at fetal HSD11B2 promoter in preeclampsia is not consistent with findings from other published studies in this area. Zhao et al. [22] found methylation levels of all studied HSD11B2 gene promoter were significantly higher in IUGR newborns. Several methodological differences

Table 2 Average methylation levels of HSD between male and female infants

\begin{tabular}{|c|c|c|c|c|c|c|}
\hline \multirow[t]{2}{*}{ Gender of neonater } & \multicolumn{2}{|c|}{ Normal pregnancy } & \multirow[t]{2}{*}{$P$ value } & \multicolumn{2}{|c|}{ Preeclampsia } & \multirow[t]{2}{*}{$P$ value } \\
\hline & Male & Female & & Male & Female & \\
\hline TOTAL N & 40 & 38 & & 22 & 21 & \\
\hline HSD9_CpG_1 & $0.13 \pm 0.032$ & $0.13 \pm 0.023$ & 0.943 & $0.12 \pm 0.025$ & $0.12 \pm 0.037$ & 0.989 \\
\hline HSD9_CpG_2 & $0.10 \pm 0.022$ & $0.11 \pm 0.016$ & 0.358 & $0.09 \pm 0.020$ & $0.10 \pm 0.016$ & 0.248 \\
\hline HSD9_CpG_3 & $0.40 \pm 0.047$ & $0.42 \pm 0.048$ & 0.087 & $0.38 \pm 0.063$ & $0.40 \pm 0.047$ & 0.481 \\
\hline HSD9 & $0.21 \pm 0.030$ & $0.22 \pm 0.020$ & 0.341 & $0.20 \pm 0.030$ & $0.21 \pm 0.030$ & 0.324 \\
\hline HSD23_CpG_1 & $0.16 \pm 0.035$ & $0.15 \pm 0.036$ & 0.103 & $0.16 \pm 0.034$ & $0.15 \pm 0.038$ & 0.417 \\
\hline HSD23_CpG_2 & $0.16 \pm 0.092$ & $0.17 \pm 0.101$ & 0.953 & $0.14 \pm 0.032$ & $0.13 \pm 0.032$ & 0.498 \\
\hline HSD23_CpG_3 & $0.15 \pm 0.060$ & $0.15 \pm 0.070$ & 0.839 & $0.12 \pm 0.056$ & $0.10 \pm 0.038$ & 0.235 \\
\hline HSD23 & $0.16 \pm 0.045$ & $0.16 \pm 0.057$ & 0.749 & $0.14 \pm 0.030$ & $0.13 \pm 0.030$ & 0.190 \\
\hline
\end{tabular}




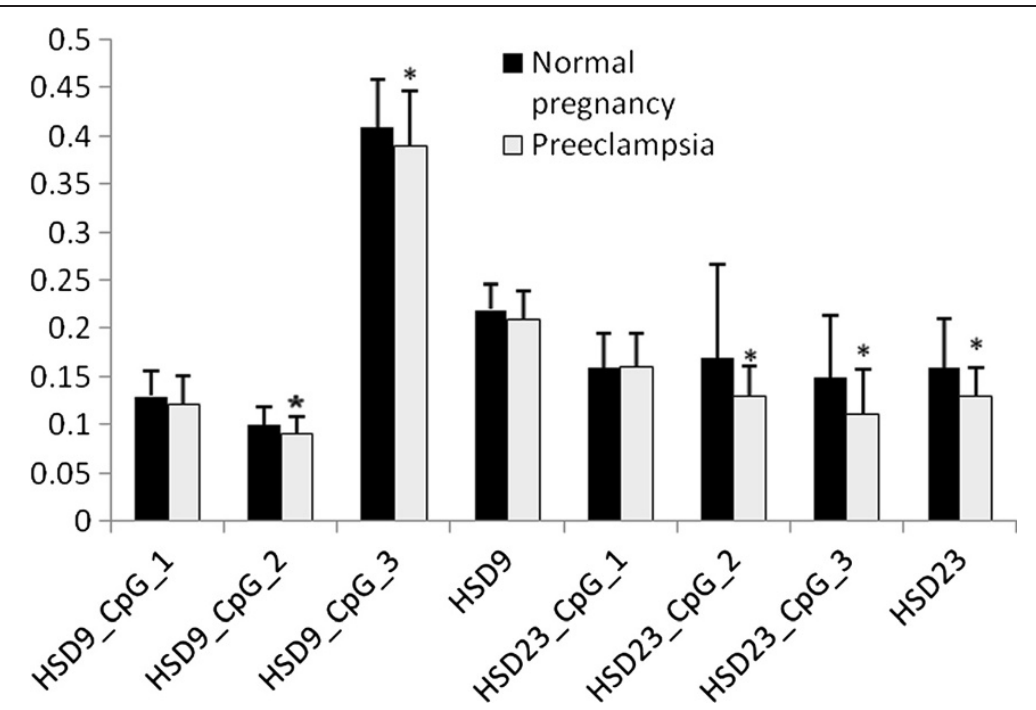

Figure 1 Average methylation levels of HSD in both normal pregancy group and preeclampsia group. There is significant differences of average methylation levels between normal pregance and preeclampsia group in HSD23_CpG2, HSD23_CpG3 and HSD23 (*P < 0.05). Data are shown as mean $\pm \mathrm{SD}$.

may help explain such inconsistencies across the two studies. We conducted a study among 43 women of preeclampsia with healthy newborn, and we took umbilical cord blood for sample. Zhao et al. employed an experimental design with 22 IUGR newborns and used pooled sample from placenta. Moreover, CpG sites in the HSD11B2 promoter regions of our sample are not the same as those of Zhao et al. As this is an emerging area of research, more work is needed to better characterize the association of preeclampsia and HSD11B2 methylation.

Numerous mechanisms have been described to control 11b-HSD2 expression and activity. Conditions compromise HSD11B2 expression and activity including enhanced availability of pro-inflammatory cytokines (TNF-a, IL-1b and Il-6), enhanced shear stress via focal adhesions and hypoxia [23-26]. Hypoxia compromises the up-regulation

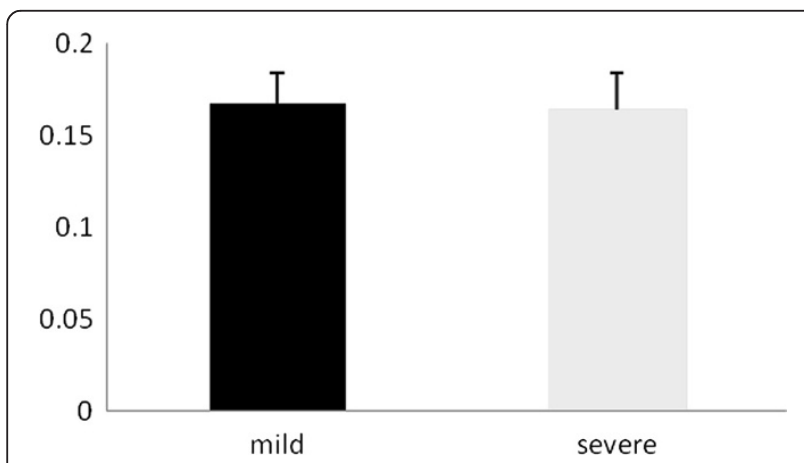

Figure 2 Comparison of methylation level between mild and severe preeclampsia. There was no significant difference in mean methylation level between mild and severe preeclampsia $(P=0.563)$. Data are shown as mean \pm SD. of HSD11B2 during the differentiation of cytotrophoblasts into syncytiotrophoblasts. These conditions were present in the placenta and fetus of preeclampsia. Epigenetic modification Methylation at CpG sites of promoter is one of the epigenetic mechanisms regulating the gene expression. DNA methylation is among the best studied epigenetic modifications and the methylation of cytosine at $\mathrm{CpG}$ dinucleotides is an important regulatory modification of genome. Other regulatory mechanisms include transcriptional regulation and posttranscriptional modification.

Generally, the low methylation at $\mathrm{CpG}$ sites of promoter results in high expression of the gene. However, a partial deficit in the activity of the enzyme 11b-HSD2 was observed in essential hypertensive patients [27] and a significantly lower level messenger RNA for HSD11B2 was found in the hypertensive patients [28]. In addition,

Table 3 Average methylation levels of HSD in both mild and severe preeclampsia group

\begin{tabular}{lccc}
\hline $\begin{array}{l}\text { Gender of } \\
\text { neonater }\end{array}$ & Mild preeclampsia & Severe preeclampsia & P value \\
\hline TOTAL N & 25 & 18 & \\
HSD9_CpG_1 & $0.12 \pm 0.022$ & $0.13 \pm 0.041$ & 0.626 \\
HSD9_CpG_2 & $0.093 \pm 0.016$ & $0.089 \pm 0.022$ & 0.505 \\
HSD9_CpG_3 & $0.400 \pm 0.044$ & $0.38 \pm 0.067$ & 0.152 \\
HSD9 & $0.21 \pm 0.024$ & $0.20 \pm 0.037$ & 0.690 \\
HSD23_CpG_1 & $0.16 \pm 0.034$ & $0.15 \pm 0.040$ & 0.753 \\
HSD23_CpG_2 & $0.13 \pm 0.032$ & $0.14 \pm 0.030$ & 0.193 \\
HSD23_CpG_3 & $0.11 \pm 0.049$ & $0.11 \pm 0.048$ & 0.818 \\
HSD23 & $0.13 \pm 0.028$ & $0.14 \pm 0.031$ & 0.672 \\
\hline
\end{tabular}


Friso et al. [29] found that elevated HSD11B2 promoter methylation was associated with hypertension developing in glucocorticoid-treated patients and essential hypertension. Taken these observations together with our findings, it is implied that hypomethylation status at the promoter of HSD11B2 may not be among the mechanisms linking intrauterine exposure to maternal preeclampsia and high risk of metabolic diseases in adulthood.

Abnormal hypermethylated or hypomethylated genes can serve as biomarkers for clinical use in early detection, classification, and prediction of treatment response. In our study, we detected the alteration in methylation status at the promoter of HSD11B2 in the offspring of preeclamptic women. We finally found that methylation levels of HSD11B2 promoter were significantly lower in preeclampsia than normal pregnancy. The positive relationship between HSD11B2 promoter methylation and infants born to preeclamptic women with the hypomethylated HSD11B2 may be a potential marker for preeclampsia. However, further studies are required to address the mechanisms leading fetal hypomethylation at HSD11B2 promoter and the significance of the hypomethylation in the development of metabolic diseases.

\section{Conclusions}

In conclusion, our study suggests that the decreased methylation at the promoter of HSD in the fetuses of preeclamptic women could be a potentially accessible biomarker for preeclampsia women. Further studies are required to explain the mechanisms of hypomethylation at HSD11B2 promoter in infants born to preeclamptic women.

\section{Competing interests}

The authors declare that they have no competing interests.

\section{Authors' contributions}

$\mathrm{WH}$ conceived of the study, participated in the design of the study, performed statistical analyses, and drafted the manuscript. XW carried out the genome-wide genotyping, targeted sequencing, quality control, performed statistical analysis, and drafted the manuscript. MD performed statistical analysis and aided in the drafting of the manuscript. $Y L$, and $W L$ participated in the design of the study and drafting the manuscript. $\mathrm{HH}$ conceived of the study, participated in its design and coordination, and helped to draft the manuscript. All authors read and approved the final manuscript.

\section{Acknowledgement}

This work was financially supported by Natural Scientific Foundation of Zhejiang Province (LY13H040004).

\section{Author details}

${ }^{1}$ Women's Hospital, School of Medicine, Zhejiang University, 1 Xueshi Road, Hangzhou, Zhejiang Province 310006, China. ${ }^{2}$ Institutes of Biomedical Sciences, Fudan University, 220 Handan Road, Shanghai, China. ${ }^{3}$ Key Laboratory of Molecular Medicine, The Ministry of Education, Department of Biochemistry and Molecular Biology, Fudan University, 220 Handan Road, Shanghai, China.
Received: 11 June 2014 Accepted: 26 August 2014

Published online: 09 September 2014

\section{References}

1. Report of the National High Blood Pressure Education Program Working Group on High Blood Pressure in Pregnancy. Am J Obstet Gynecol 2000, 183:S1-S22

2. Rich-Edwards JW, Grizzard TA: Psychosocial stress and neuroendocrine mechanisms in preterm delivery. Am J Obstet Gynecol 2005, 192:S30-S35.

3. Curhan GC, Chertow GM, Willett WC, Spiegelman D, Colditz GA, Colditz GA, Manson JE, Speizer FE, Stampfer MJ: Birth weight and adult hypertension and obesity in women. Circulation 1996, 94:1310-1315.

4. Barker DJ, Hales CN, Fall CH, Osmond C, Phipps K, Clark PM: Type 2 (non-insulin-dependent) diabetes mellitus, hypertension and hyperlipidaemia (syndrome X): relation to reduced fetal growth. Diabetologia 1993, 36:62-67.

5. Serman L, Vlahovic M, Sijan M, Bulic-Jakus F, Serman A, Sincic N, Matijevic R, Juric-Lekic $G$, Katusic A: The impact of 5-azacytidine on placental weight, glycoprotein pattern and proliferating cell nuclear antigen expression in rat placenta. Placenta 2007, 28:803-811.

6. He J, Zhang A, Fang M, Fang R, Ge J, Jiang Y, Zhang H, Han C, Ye X, Yu D, Huang $H$, Liu Y, Dong M: Methylation levels at IGF2 and GNAS DMRs in infants born to preeclamptic pregnancies. BMC Genomics 2013, 14:472.

7. American College of O, Gynecologists: ACOG practice bulletin. Management of infertility caused by ovulatory dysfunction. Number 34, February 2002. American College of Obstetricians and Gynecologists. Int J Gynaecol Obstet 2002, 77:177-188.

8. Schoof E, Girstl M, Frobenius W, Kirschbaum M, Dorr HG, Rascher W, Dotsch J: Decreased gene expression of 11 beta-hydroxysteroid dehydrogenase type 2 and 15-hydroxyprostaglandin dehydrogenase in human placenta of patients with preeclampsia. J Clin Endocrinol Metab 2001, 86:1313-1317.

9. He P, Chen Z, Sun Q, Li Y, Gu H, Ni X: Reduced expression of 11 beta-hydroxysteroid dehydrogenase type 2 in preeclamptic placentas is associated with decreased PPARgamma but increased PPARalpha expression. Endocrinology 2014, 155:299-309.

10. Hardy DB, Yang K: The expression of 11 beta-hydroxysteroid dehydrogenase type 2 is induced during trophoblast differentiation: effects of hypoxia. J Clin Endocrinol Metab 2002, 87:3696-3701.

11. Alfaidy N, Gupta S, DeMarco C, Caniggia I, Challis JR: Oxygen regulation of placental 11 b-hydroxysteroid dehydrogenase 2: physiological and pathological implications. J Clin Endocrinol 2002, 87:4797-4805.

12. Benediktsson $R$, Calder AA, Edwards CR, Seckl JR: Placental 11b-hydroxysteroid dehydrogenase: a key regulator of fetal glucocorticoid exposure. Clin Endocrinol (Oxf) 1997, 46:161-166.

13. Goland RS, Jozak S, Warren WB, Conwell IM, Stark RI, Tropper PJ: Elevated levels of umbilical cord plasma corticotropin-releasing hormone in growth-retarded fetuses. J Clin Endocrinol Metab 1993, 77:1174-1179.

14. Goland RS, Tropper PJ, Warren WB, Stark RI, Jozak SM, Conwell IM: Concentrations of corticotrophin-releasing hormone in the umbilical-cord blood of pregnancies complicated by pre-eclampsia. Reprod Fertil Dev 1995, 7:1227-1230.

15. Seckl JR, Meaney MJ: Glucocorticoid programming. Ann N Y Acad Sci 2004, 1032:63-84.

16. Meaney MJ, Szyf M, Seckl JR: Epigenetic mechanisms of perinatal programming of hypothalamic-pituitary-adrenal function and health. Trends Mol Med 2007, 13:269-277.

17. Jaquiery $\mathrm{AL}$, Oliver MH, Bloomfield FH, Connor KL, Challis JR, Harding JE: Fetal exposure to excess glucocorticoid is unlikely to explain the effects of periconceptional undernutrition in sheep. J Physio/ 2006, 572:109-118.

18. Dave-Sharma S, Wilson RC, Harbison MD, Newfield R, Azar MR, Krozowski ZS, Funder JW, Shackleton CH, Bradlow HL, Wei JQ, Hertecant J, Moran A, Neiberger RE, Balfe JW, Fattah A, Daneman D, Akkurt HI, De Santis C, New Ml: Examination of genotype and phenotype relationships in 14 patients with apparent mineralocorticoid excess. J Clin Endocrinol 1998, 83:2244-2254.

19. Blasco MJ, Lopez Bernal A, Turnbull AC: 11 beta-Hydroxysteroid dehydrogenase activity of the human placenta during pregnancy. Horm Metab Res 1986, 18:638-641.

20. Stewart PM, Murry BA, Mason Jl: Type 211 beta-hydroxysteroid dehydrogenase in human fetal tissues. J Clin Endocrinol Metab 1994, 78:1529-1532.

21. Brown RW, Chapman KE, Kotelevtsev Y, Yau JL, Lindsay RS, Brett L, Leckie C, Murad P, Lyons V, Mullins JJ, Edwards CR, SeckI JR: Cloning and production 
of antisera to human placental 11 beta-hydroxysteroid dehydrogenase type 2. Biochem J 1996, 313(Pt 3):1007-1017.

22. Zhao Y, Gong X, Chen L, Li L, Liang Y, Chen S, Zhang Y: Site-specific methylation of placental HSD11B2 gene promoter is related to intrauterine growth restriction. Eur J Hum Genet 2014, 22:734-740.

23. Lanz B, Kadereit B, Ernst S, Shojaati K, Causevic M, Frey BM, Frey FJ, Mohaupt MG: Angiotensin II regulates 11 beta-hydroxysteroid dehydrogenase type 2 via AT2 receptors. Kidney Int 2003, 64:970-977.

24. Heiniger CD, Kostadinova RM, Rochat MK, Serra A, Ferrari P, Dick B, Frey BM, Frey FJ: Hypoxia causes down-regulation of 11 beta-hydroxysteroid dehydrogenase type 2 by induction of Egr-1. FASEB J 2003, 17:917-919.

25. Lanz CB, Causevic M, Heiniger C, Frey FJ, Frey BM, Mohaupt MG:

Fluid shear stress reduces 11ss-hydroxysteroid dehydrogenase type 2 . Hypertension 2001, 37:160-169.

26. Kossintseva I, Wong S, Johnstone E, Guilbert L, Olson DM, Mitchell BF: Proinflammatory cytokines inhibit human placental 11 betahydroxysteroid dehydrogenase type 2 activity through $\mathrm{Ca} 2+$ and CAMP pathways. Am J Physiol Endocrinol Metab 2006, 290:E282-E288.

27. Campino C, Carvajal CA, Cornejo J, San Martin B, Olivieri O, Guidi G, Faccini G, Pasini F, Sateler J, Baudrand R, Mosso L, Owen Gl, Kalergis AM, Padilla O, Fardella CE: 11 beta-Hydroxysteroid dehydrogenase type-2 and type-1 (11beta-HSD2 and 11beta-HSD1) and 5beta-reductase activities in the pathogenia of essential hypertension. Endocrine 2010, 37:106-114.

28. Agalou S, Butt AN, Chowienczyk P, Swaminathan R: Hypertension and circulating mRNA for 11beta-hydroxysteroid dehydrogenase type II. Ann N Y Acad Sci 2008, 1137:290-295.

29. Friso S, Pizzolo F, Choi SW, Guarini P, Castagna A, Ravagnani V, Carletto A, Pattini P, Corrocher R, Olivieri O: Epigenetic control of 11 beta-hydroxysteroid dehydrogenase 2 gene promoter is related to human hypertension. Atherosclerosis 2008, 199:323-327.

doi:10.1186/s12863-014-0096-5

Cite this article as: Hu et al: Alteration in methylation level at $11 \beta$-hydroxysteroid dehydrogenase type 2 gene promoter in infants born to preeclamptic women. BMC Genetics 2014 15:96.

\section{Submit your next manuscript to BioMed Central and take full advantage of:}

- Convenient online submission

- Thorough peer review

- No space constraints or color figure charges

- Immediate publication on acceptance

- Inclusion in PubMed, CAS, Scopus and Google Scholar

- Research which is freely available for redistribution 